\title{
Entire solutions of certain type of difference equations
}

\author{
Nana Liu', Weiran Lü ${ }^{1 *}$, Tiantian Shen ${ }^{1}$ and Chungchun Yang ${ }^{2}$
}

\footnotetext{
"Correspondence: luwr@upc.edu.cn ${ }^{1}$ Department of Mathematics, China University of Petroleum, Qingdao, 266580, P.R. China

Full list of author information is available at the end of the article
}

\begin{abstract}
In this paper, we shall study the conditions regarding the existence of transcendental entire solutions of certain type of difference equations. Our results are either supplements to some results obtained recently, or are relating to the conjecture raised in Yang and Laine (Proc. Jpn. Acad., Ser. A, Math. Sci. 86:10-14, 2010). Finally, two relevant conjectures are posed for further studies.
\end{abstract}

MSC: 39B32; 34M05; 30D35

Keywords: transcendental entire solution; difference equation; difference-differential polynomial; Nevanlinna theory

\section{Introduction, notations and main results}

Let $f$ denote a transcendental entire or meromorphic function. Assuming the reader is familiar with the basics of Nevanlinna's value distribution theory, we shall adopt the standard notations associated with the theory, such as the characteristic function $T(r, f)$, the counting function of the poles $N(r, f)$, and the proximity function $m(r, f)$ (see, e.g., [1, 2]).

Among many interesting applications of the Nevanlinna theory, there are studies on the growth and existence of entire or meromorphic solutions of various types of non-linear differential equations, and one can find prototypes for such equations, e.g., in [3-5] and [6]. Recently, the Nevanlinna theory has been applied to study types of non-linear difference equations, see, e.g., [7, 8]. Now, we shall utilize Clunie type of theorems for differencedifferential polynomials to study some non-linear difference equations of more general forms and to obtain some improvements of or supplements to [7, 9], and [10].

Notations Given a meromorphic function $f$, recall that $\alpha \neq \equiv 0, \infty$ is a small function with respect to $f$, if $T(r, \alpha)=S(r, f)$, where $S(r, f)$ denotes any quantity satisfying $S(r, f)=$ $o\{T(r, f)\}$ as $r \rightarrow+\infty$, possibly outside a set of $r$ of finite linear measure. For a constant $c \in \mathbb{C}, f(z+c)$ is called a shift of $f$. As for a difference product, we mean a difference monomial of type $\prod_{j=1}^{k} f\left(z+c_{j}\right)^{n_{j}}$, where $c_{1}, \ldots, c_{k}$ are complex constants and $n_{1}, \ldots, n_{k}$ are natural numbers.

Definition 1.1 A difference polynomial, respectively, a difference-differential polynomial, in $f$ is a finite sum of difference products of $f$ and its shifts, respectively, of products of $f$, derivatives of $f$ and of their shifts, with all the coefficients of these monomials being small functions of $f$.

○2014 Liu et al.; licensee Springer. This is an Open Access article distributed under the terms of the Creative Commons Attribution License (http://creativecommons.org/licenses/by/2.0), which permits unrestricted use, distribution, and reproduction in any medium, provided the original work is properly cited. 
Consider a transcendental meromorphic function $f(z)$ and let

$$
P(z, f)=\sum_{l=1}^{n} a_{l}(z) \prod_{j=0}^{k}\left\{f^{(j)}\left(z+c_{l j}\right)\right\}^{n_{l j}},
$$

where $a_{l}(z)(l=1,2, \ldots, n)$ are small functions of $f$, and $c_{l j}(l=1,2, \ldots, n ; j=0,1, \ldots, k)$ are complex constants, and $n_{l j}(l=1,2, \ldots, n ; j=0,1, \ldots, k)$ are natural numbers.

Two terms $a_{l}(z) \prod_{j=0}^{k}\left\{f^{(j)}\left(z+c_{l j}\right)\right\}^{n_{l j}}$ and $a_{l^{\prime}}(z) \prod_{j=0}^{k}\left\{f^{(j)}\left(z+c_{l j}\right)\right\}^{n_{l^{\prime} j}}$ are called similar, if

$$
n_{l j}=n_{l^{\prime} j} \quad(j=0,1, \ldots, k) .
$$

Group together similar terms of $P(z, f)$, if necessary. In the following, we assume that no two terms of $P(z, f)$ are similar and that $a_{l}(z) \not \equiv 0(l=1,2, \ldots, n)$.

Definition 1.2 We define the total degree $d$ of $P(z, f)$

$$
d:=d(P(z, f))=\max _{1 \leq l \leq n}\left\{\sum_{j=0}^{k} n_{l j}\right\} .
$$

For the sake of simplicity, we let $\Delta f(z)=f(z+1)-f(z), \Delta^{n} f(z)=\Delta\left(\Delta^{n-1} f(z)\right)(n \geq 2)$.

Yang and Laine [10] considered the following difference equation and proved it.

Theorem A A non-linear difference equation

$$
f^{3}(z)+q(z) f(z+1)=c \sin b z
$$

where $q(z)$ is a non-constant polynomial and $b, c \in \mathbb{C}$ are nonzero constants, does not admit entire solutions of finite order. If $q(z)=q$ is a nonzero constant, then the above equation possesses three distinct entire solutions of finite order, provided that $b=3 n \pi$ and $q^{3}=(-1)^{n+1} c^{2} 27 / 4$ for a nonzero integer $n$.

Now, we shall substitute $f(z+1)$ by $\Delta f(z)$ in Theorem A and prove the following results.

Theorem 1.1 Let $n \geq 4$ be an integer, $q(z)$ be a polynomial, and $p_{1}, p_{2}, \alpha_{1}, \alpha_{2}$ be nonzero constants such that $\alpha_{1} \neq \alpha_{2}$. If there exists some entire solution $f$ of finite order to (1.1) below

$$
f^{n}(z)+q(z) \Delta f(z)=p_{1} \mathrm{e}^{\alpha_{1} z}+p_{2} \mathrm{e}^{\alpha_{2} z},
$$

then $q(z)$ is a constant, and one of the following relations holds:

(1) $f(z)=c_{1} \mathrm{e}^{\frac{\alpha_{1}}{n} z}$, and $c_{1}\left(\mathrm{e}^{\alpha_{1} / n}-1\right) q=p_{2}, \alpha_{1}=n \alpha_{2}$,

(2) $f(z)=c_{2} \mathrm{e}^{\frac{\alpha_{2}}{n} z}$, and $c_{2}\left(\mathrm{e}^{\alpha_{2} / n}-1\right) q=p_{1}, \alpha_{2}=n \alpha_{1}$,

where $c_{1}, c_{2}$ are constants satisfying $c_{1}^{n}=p_{1}, c_{2}^{n}=p_{2}$. 
Corollary 1.1 Let $n \geq 4$ be an integer, $q(z) \not \equiv 0$ be a polynomial, and $p, \alpha$ be nonzero constants. Then the non-linear difference equation

$$
f^{n}(z)+q(z) \Delta f(z)=p \mathrm{e}^{\alpha z}
$$

has no transcendental entire solutions of finite order provided that $\frac{\alpha}{n} \neq 2 k \pi \mathrm{i}$, where $k$ is an integer.

By some further analysis, we can derive the following result.

Theorem 1.2 A non-linear difference equation

$$
f^{3}(z)+q(z) \Delta^{3} f(z)=c \sin b z
$$

where $q(z)$ is a non-constant polynomial and $b, c \in \mathbb{C}$ are nonzero constants, does not admit entire solutions of finite order. If $q(z)=q$ is a nonzero constant, then (1.2) possesses solutions of the form $f(z)=c_{1} \mathrm{e}^{\frac{b \mathrm{i}}{3} z}+c_{2} \mathrm{e}^{-\frac{b \mathrm{i}}{3} z}, c_{1}^{3}=-c_{2}^{3}=\frac{c}{2 \mathrm{i}}$, provided that $b=3 n \pi, n$ is an odd number, $q^{3}=\frac{27}{2,048} c^{2}$ or $b=6 n \pi \pm \pi, q^{3}=-\frac{27}{4} c^{2}$.

Examples In the special case of

$$
f^{3}(z)-\frac{3}{8} \Delta^{3} f(z)=2 \mathrm{i} \sin 3 \pi z
$$

a finite order entire solution is $f(z)=2 \mathrm{i} \sin \pi z=\mathrm{e}^{\mathrm{i} \pi z}-\mathrm{e}^{-\mathrm{i} \pi z}$.

$$
\text { And } f^{3}(z)+3 \Delta^{3} f(z)=2 \mathrm{i} \sin 7 \pi z \text { has the entire solution } f(z)=\mathrm{e}^{\frac{7 \pi}{3} \mathrm{i} z}-\mathrm{e}^{-\frac{7 \pi}{3} \mathrm{i} z} \text {. }
$$

Corollary 1.2 The non-linear difference equation

$$
f^{n}+q \Delta f(z)=c \sin b z
$$

where $q$ is a nonzero constant, possesses solutions of the form $f=c_{1} \mathrm{e}^{\frac{b \mathrm{i}}{3} z}+c_{2} \mathrm{e}^{-\frac{b \mathrm{i}}{3}}$, if and only if $n=3$.

Theorem B ([10], Theorem 2.4) Let $p, q$ be polynomials, then a non-linear difference equation

$$
f^{2}(z)+q(z) f(z+1)=p(z)
$$

has no transcendental entire solutions of finite order.

We shall modify the equation in Theorem B above and derive the following result.

Theorem 1.3 Let $l \geq 2, n \geq 1$ be integers, $a(z)$ and $b(z)$ be meromorphic functions such that $T(r, a)=\lambda_{1} T(r, f)+S(r, f), T(r, b)=\lambda_{2} T(r, f)+S(r, f)$, where non-negative numbers $\lambda_{1}, \lambda_{2}$ satisfy $\lambda_{1}+\lambda_{2}<1$. Then

$$
f^{l}(z)+a(z) \Delta^{n} f(z)=b(z)
$$

has no transcendental entire solutions of hyper-order $\sigma_{2}(f)<1$. 


\section{Preliminaries}

In order to prove our conclusions, we need some lemmas.

Lemma 2.1 ([7]) Let $f$ be a transcendental meromorphic solution of finite order $\rho$ of a difference equation of the form

$$
H(z, f) P(z, f)=Q(z, f)
$$

where $H(z, f), P(z, f), Q(z, f)$ are difference polynomials in $f$ such that the total degree of $H(z, f)$ in $f$ and its shifts is $n$, and that the corresponding total degree of $Q(z, f)$ is $\leq n$. If $H(z, f)$ contains just one term of maximal total degree, then for any $\varepsilon>0$,

$$
m(r, P(z, f))=O\left(r^{\rho-1+\varepsilon}\right)+S(r, f)
$$

possibly outside of an exceptional set of finite logarithmic measure.

Remark 2.1 The following result is a Clunie type lemma [11] for the difference-differential polynomials of a meromorphic function $f$. It can be proved by applying Lemma 2.1 with a similar reasoning as in [10] and stated as follows.

Let $f(z)$ be a meromorphic function of finite order, and let $P(z, f), Q(z, f)$ be two difference-differential polynomials of $f$. If $f^{n} P(z, f)=Q(z, f)$ holds and if the total degree of $Q(z, f)$ in $f$ and its derivatives and their shifts is $\leq n$, then $m(r, P(z, f))=S(r, f)$.

Lemma 2.2 ([12]) Suppose that $m, n$ are positive integers satisfying $\frac{1}{m}+\frac{1}{n}<1$. Then there exist no transcendental entire solutions $f$ and $g$ satisfying the equation $a f^{n}+b g^{m}=1$, with $a, b$ being small functions off and $g$, respectively.

Lemma 2.3 ([13]) Assume that $c \in \mathbb{C}$ is a nonzero constant, $\alpha$ is a non-constant meromorphic function. Then the differential equation $f^{2}+\left(c f^{(n)}\right)^{2}=\alpha$ has no transcendental meromorphic solutions satisfying $T(r, \alpha)=S(r, f)$.

Lemma 2.4 ([14, 15]) Let $f$ be a transcendental meromorphic function of finite order $\rho$, then for any complex numbers $c_{1}, c_{2}$ and for each $\varepsilon>0, m\left(r, \frac{f\left(z+c_{1}\right)}{f\left(z+c_{2}\right)}\right)=O\left(r^{\rho-1+\varepsilon}\right)$.

References [16] and [17] further pointed out the following.

Remark 2.2 If $f$ is a non-constant finite order meromorphic function and $c \in \mathbb{C}$, then

$$
m\left(r, \frac{f(z+c)}{f(z)}\right)=S(r, f)
$$

outside of a possible exceptional set with finite logarithmic measure.

We also know that Remark 2.2 has been improved by Halburd et al. [18]. They proved that (2.2) is also true when $f$ is a meromorphic function of hyper-order $\sigma_{2}(f)<1$. 


\section{Proof of Theorem 1.1}

Suppose that $f$ is a transcendental entire solution of finite order to (1.1). By differentiating both sides of (1.1), we have

$$
n f^{n-1} f^{\prime}+(q(z) \Delta f(z))^{\prime}=\alpha_{1} p_{1} \mathrm{e}^{\alpha_{1} z}+\alpha_{2} p_{2} \mathrm{e}^{\alpha_{2} z} .
$$

From (1.1) and (3.1), we obtain

$$
\begin{aligned}
& \alpha_{2} f^{n}+\alpha_{2} q(z) \Delta f(z)-n f^{n-1} f^{\prime}-(q(z) \Delta f(z))^{\prime}=\left(\alpha_{2}-\alpha_{1}\right) p_{1} \mathrm{e}^{\alpha_{1} z}, \\
& \alpha_{1} f^{n}+\alpha_{1} q(z) \Delta f(z)-n f^{n-1} f^{\prime}-(q(z) \Delta f(z))^{\prime}=\left(\alpha_{1}-\alpha_{2}\right) p_{2} \mathrm{e}^{\alpha_{2} z} .
\end{aligned}
$$

Differentiating (3.2) yields

$$
\begin{aligned}
& n \alpha_{2} f^{n-1} f^{\prime}+\alpha_{2}(q(z) \Delta f(z))^{\prime}-n(n-1) f^{n-2}\left(f^{\prime}\right)^{2}-n f^{n-1} f^{\prime \prime}-(q(z) \Delta f(z))^{\prime \prime} \\
& \quad=\alpha_{1}\left(\alpha_{2}-\alpha_{1}\right) p_{1} \mathrm{e}^{\alpha_{1} z} .
\end{aligned}
$$

It follows from (3.2) and (3.4) that

$$
f^{n-2} \varphi=T(f)
$$

where

$$
\begin{aligned}
& \varphi=\alpha_{1} \alpha_{2} f^{2}-n\left(\alpha_{1}+\alpha_{2}\right) f^{\prime} f+n(n-1)\left(f^{\prime}\right)^{2}+n f^{\prime \prime} f, \\
& T(f)=-\alpha_{1} \alpha_{2} q(z) \Delta f(z)+\left(\alpha_{1}+\alpha_{2}\right)(q(z) \Delta f(z))^{\prime}-(q(z) \Delta f(z))^{\prime \prime} .
\end{aligned}
$$

Next, we shall prove $\varphi \equiv 0$. In fact, since $T$ is a difference-differential polynomial in $f$, and its degree at most 1. By (3.5) and Remark 2.1 after Lemma 2.1, we have $m(r, \varphi)=S(r, f)$, and $T(r, \varphi)=S(r, f)$. On the other hand, we can rewrite (3.5) as $f^{n-3} f \varphi=T(f)$, which implies $m(r, f \varphi)=S(r, f)$, and $T(r, f \varphi)=S(r, f)$. If $\varphi \not \equiv 0$, then $T(r, f)=T\left(r, \frac{f \varphi}{\varphi}\right)=S(r, f)$, and this is impossible. Hence $\varphi \equiv 0$, and $T \equiv 0$, i.e.

$$
-\alpha_{1} \alpha_{2} q(z) \Delta f(z)+\left(\alpha_{1}+\alpha_{2}\right)(q(z) \Delta f(z))^{\prime}-(q(z) \Delta f(z))^{\prime \prime} \equiv 0 .
$$

If $q(z) \Delta f(z) \equiv 0$, then (1.1) can be rewritten as

$$
\frac{1}{p_{1}}\left(f \mathrm{e}^{-\frac{\alpha_{1}}{n} z}\right)^{n}-\frac{p_{2}}{p_{1}}\left(\mathrm{e}^{\frac{1}{3}\left(\alpha_{2}-\alpha_{1}\right) z}\right)^{3}=1,
$$

which is impossible by Lemma 2.2. Thus, $q(z) \Delta f(z) \not \equiv 0$. It is easily seen from $\alpha_{1} \neq \alpha_{2}$ that

$$
\alpha_{1} q(z) \Delta f(z)-(q(z) \Delta f(z))^{\prime} \equiv 0
$$

and

$$
\alpha_{2} q(z) \Delta f(z)-(q(z) \Delta f(z))^{\prime} \equiv 0
$$

cannot hold simultaneously. 
First of all, we assume that $\alpha_{2} q(z) \Delta f(z)-(q(z) \Delta f(z))^{\prime} \not \equiv 0$, then (3.6) gives

$$
\alpha_{2} q(z) \Delta f(z)-(q(z) \Delta f(z))^{\prime}=A \mathrm{e}^{\alpha_{1} z},
$$

where $A$ is a nonzero constant. Substituting the above expression into (3.2), we obtain

$$
f^{n-1}\left(\alpha_{2} f-n f^{\prime}\right)=\frac{\left(\alpha_{2}-\alpha_{1}\right) p_{1}-A}{A}\left(\alpha_{2} q(z) \Delta f(z)-(q(z) \Delta f(z))^{\prime}\right) .
$$

Again, Lemma 2.1 shows that $\alpha_{2} f-n f^{\prime} \equiv 0,\left(\alpha_{2}-\alpha_{1}\right) p_{1}=A$. So

$$
f^{n}=B \mathrm{e}^{\alpha_{2} z},
$$

where $B$ is a constant.

Substituting (3.7) into (1.1), we find

$$
\left(1-\frac{p_{2}}{B}\right) f^{n}=\frac{p_{1}\left(\alpha_{2} q(z) \Delta f(z)-(q(z) \Delta f(z))^{\prime}\right)}{A}-q(z) \Delta f(z) .
$$

If $B \neq p_{2}$, by Lemma 2.1, we get $T(r, f)=m(r, f)=S(r, f)$, which is absurd. So $B=p_{2}$, and $f=c_{2} \mathrm{e}^{\frac{\alpha_{2}}{n} z}=c_{2} \mathrm{e}^{\alpha_{1} z}, c_{2}^{n}=B=p_{2}, c_{2}\left(\mathrm{e}^{\alpha_{2} / n}-1\right) q=p_{1}$.

If $\alpha_{1} q(z) \Delta f(z)-(q(z) \Delta f(z))^{\prime} \not \equiv 0$, by (3.3) and using similar arguments as above, we can derive $f=c_{1} \mathrm{e}^{\frac{\alpha_{1}}{n} z}=c_{1} \mathrm{e}^{\alpha_{2} z}, c_{1}^{n}=p_{1}, c_{1}\left(\mathrm{e}^{\alpha_{1} / n}-1\right) q=p_{2}$.

This completes the proof of Theorem 1.1.

Remark 3.1 Suggested by the referee, one can also derive the conclusions of Theorem 1.1 when $\varphi \equiv 0$.

In fact, since $\varphi$ in (3.5) vanishes identically, dividing with $f^{2}$, and recalling $f^{\prime \prime} \mid f=\left(f^{\prime} \mid f\right)^{\prime}+$ $\left(f^{\prime} \mid f\right)^{2}$, we get the Riccati equation

$$
t^{\prime}+n t^{2}-\left(\alpha_{1}+\alpha_{2}\right) t+\alpha_{1} \alpha_{2} / n=0
$$

where $t:=f^{\prime} \mid f$. This equation has two constant solutions, $t=\alpha_{1} / n, t=\alpha_{2} / n$. By Corollary 5.2 in the paper by Bank et al. [19], all other meromorphic solutions are of infinite order. And from this one can obtain the two entire solutions of exponential type.

\section{Proof of Theorem 1.2}

Suppose that $f$ is a transcendental entire solution of finite order to (1.2), differentiating (1.2) results in

$$
3 f^{2} f^{\prime}+q^{\prime}(z) \Delta^{3} f(z)+q(z)\left(f^{\prime}(z+3)-3 f^{\prime}(z+2)+3 f^{\prime}(z+1)-f^{\prime}(z)\right)=b c \cos b z .
$$

Combining (1.2) and (4.1), we obtain

$$
f^{4}\left[9\left(f^{\prime}\right)^{2}+b^{2} f^{2}\right]=T_{4}(f)
$$

where $T_{4}(f)$ is a difference-differential polynomial of $f$, and its total degree at most 4 . 
If $T_{4}(f) \equiv 0$, it follows from (4.2) that $9\left(f^{\prime}\right)^{2}+b^{2} f^{2} \equiv 0$, then $f^{\prime}= \pm \frac{b \mathrm{i}}{3} f$, and there exists a nonzero constant $c$ such that $f=c \mathrm{e}^{ \pm \frac{b \mathrm{i}}{3}}$. Substituting the expression of $f$ into (1.2), we arrive at a contradiction. Therefore, $T_{4}(f) \not \equiv 0$. Set $\beta=9\left(f^{\prime}\right)^{2}+b^{2} f^{2}$, then Lemma 2.1 shows that $m(r, \beta)=S(r, f)$, i.e. $\beta$ is a small function of $f$. Moreover, from Lemma 2.3 , it is easy to see that $\beta$ is a constant. By differentiating both sides of $\beta=9\left(f^{\prime}\right)^{2}+b^{2} f^{2}$, we get

$$
f^{\prime \prime}+\left(\frac{b}{3}\right)^{2} f=0
$$

It follows from (4.3) that

$$
f=c_{1} \mathrm{e}^{\frac{b \mathrm{i}}{3} z}+c_{2} \mathrm{e}^{-\frac{b \mathrm{i}}{3} z}
$$

where $c_{1}, c_{2}$ are constants. Substituting (4.4) into (1.2), we have

$$
a_{1} w^{6}+a_{2} w^{4}+a_{3} w^{2}+a_{4}=0
$$

where $w=\mathrm{e}^{\frac{b \mathrm{i}}{3} z}, a_{1}=c_{1}^{3}-\frac{c}{2 \mathrm{i}}, a_{2}=3 c_{1}^{2} c_{2}+c_{1} q(z) \mathrm{e}^{b \mathrm{i}}-3 q(z) c_{1} \mathrm{e}^{\frac{2 b \mathrm{i}}{3}}+3 q(z) c_{1} \mathrm{e}^{\frac{b \mathrm{i}}{3}}-c_{1} q(z), a_{3}=$ $3 c_{1} c_{2}^{2}+c_{2} q(z) \mathrm{e}^{-b \mathrm{i}}-3 q(z) c_{2} \mathrm{e}^{-\frac{2 b \mathrm{i}}{3}}+3 q(z) c_{2} \mathrm{e}^{-\frac{b \mathrm{i}}{3}}-c_{2} q(z), a_{4}=c_{2}^{3}+\frac{c}{2 \mathrm{i}}$.

Since $w$ is transcendental, we must have $a_{1}=a_{2}=a_{3}=a_{4}=0$. It follows from $a_{2}=a_{3}=0$ that

$$
\left(\mathrm{e}^{\frac{b \mathrm{i}}{3}}-1\right)^{3}=\left(\mathrm{e}^{-\frac{b \mathrm{i}}{3}}-1\right)^{3} .
$$

Let $v=\mathrm{e}^{\frac{b \mathrm{i}}{3}}$, then from (4.5) we get

$$
v-1=u_{k}\left(\frac{1}{v}-1\right), \quad k=1,2,3
$$

where $u_{1}=1, u_{2}=\frac{-1+\sqrt{3} \mathbf{i}}{2}, u_{3}=\frac{-1-\sqrt{3} \mathbf{i}}{2}$.

Now we distinguish three cases to discuss.

Case 1. Let $u_{k}=1$, we conclude $\mathrm{e}^{\frac{2 b \mathrm{i}}{3}}=1=\mathrm{e}^{2 n \pi \mathrm{i}}$, so $b=3 n \pi$, which and $a_{2}=0$ imply that

$$
3 c_{1} c_{2}+q(z)\left(v^{3}-3 v^{2}+3 v-1\right)=0
$$

and $3 c_{1} c_{2}=4 q(z)\left(1-(-1)^{n}\right)$, where $c_{1} \neq 0, c_{2} \neq 0$ are constants. Therefore $n$ is odd and $q^{3}=\frac{27}{2,048} c^{2}$.

Case 2. Assume that $u_{k}=\frac{-1+\sqrt{3} \mathrm{i}}{2}$; by a similar method as Case 1 , we get $b=6 n \pi$ or $b=$ $6 n \pi-\pi$.

If $b=6 n \pi$, combining with $a_{2}=0$, we find $c_{1} c_{2}=0$, which is a contradiction.

If $b=6 n \pi-\pi$, substituting this expression of $b$ into $a_{2}=0$, we have $q^{3}=-\frac{27}{4} c^{2}$.

Case 3. While $u_{k}=\frac{-1-\sqrt{3} \mathrm{i}}{2}$, using a similar way as above, we get $b=6 n \pi$ or $b=6 n \pi+\pi$. If $b=6 n \pi$, we can get a contradiction, hence $b=6 n \pi+\pi$, and $q^{3}=-\frac{27}{4} c^{2}$.

This completes the proof of Theorem 1.2. 


\section{Proof of Theorem 1.3}

Suppose that $f$ is a transcendental entire solutions of hyper-order $\sigma_{2}(f)<1$ to (1.3). Without loss of generality, we assume that $a \not \equiv 0$. Remark 2.2 after Lemma 2.4 and (1.3) will give

$$
\begin{aligned}
\operatorname{lm}(r, f) & =m\left(r, b-a \Delta^{n} f(z)\right) \\
& \leq m(r, f)+O\left(r^{\rho-1+\varepsilon}\right)+\left(\lambda_{1}+\lambda_{2}\right) T(r, f)+S(r, f) .
\end{aligned}
$$

So

$$
(l-1) T(r, f)=(l-1) m(r, f) \leq O\left(r^{\rho-1+\varepsilon}\right)+\left(\lambda_{1}+\lambda_{2}\right) T(r, f)+S(r, f) .
$$

For $l \geq 2, \lambda_{1}+\lambda_{2}<1$, we get a contradiction, thus (1.3) has no transcendental entire solutions of hyper-order $\sigma_{2}(f)<1$.

This also completes the proof of Theorem 1.3.

\section{Final remark and conjectures}

The current Clunie types of theorems regarding difference-differential polynomials are mainly useful or effective to deal with problems relating to entire or meromorphic solutions of finite order for certain types of difference-differential equations. Thus, it is very natural for us to pose the following two conjectures, for further studies.

Conjecture 6.1 There are no entire solutions of infinite order for any equations (1.1), (1.2), and (1.3).

Conjecture 6.2 Equations of the forms

$$
f^{n}(z)+P\left(z, f(z), f\left(z+c_{1}\right), \ldots, f\left(z+c_{k}\right)\right)=q_{1}(z) \mathrm{e}^{\alpha_{1} z}+q_{2}(z) \mathrm{e}^{\alpha_{2} z}
$$

have no entire solutions of infinite order, where $P\left(z, f(z), f\left(z+c_{1}\right), \ldots, f\left(z+c_{k}\right)\right)$ is a difference polynomial, $q_{1}, q_{2}$ are polynomials, $n \geq 2$ and $k \geq 1$ are integers, and $\alpha_{1}, \alpha_{2}, c_{j}$ $(j=1,2, \ldots, k)$ are constants.

Competing interests

The authors declare that they have no competing interests.

Authors' contributions

All authors drafted the manuscript, read and approved the final manuscript.

Author details

${ }^{1}$ Department of Mathematics, China University of Petroleum, Qingdao, 266580, P.R. China. ${ }^{2}$ Department of Mathematics, Nanjing University, Nanjing, 210093, P.R. China.

Acknowledgements

The authors would like to thank the referee for his/her reading of the original version of the manuscript with valuable suggestions and comments. 


\section{References}

1. Hayman, WK: Meromorphic Functions. Clarendon, Oxford (1964)

2. Yi, HX, Yang, CC: Uniqueness Theory of Meromorphic Functions. Science Press, Beijing (1995)

3. Yosida, K: On algebroid solutions of ordinary equations. Jpn. J. Math. 10, 199-208 (1934)

4. Wittich, H: Eindeutige Lösungen der Differentialgleichungen $\omega^{\prime \prime}=P(z, \omega)$. Math. Ann. 125, 355-365 (1953)

5. Wittich, H: Zur Theorie linearer Differentialgleichungen im Komplexen. Ann. Acad. Sci. Fenn. Ser. A I, vol. 379 (1966)

6. Yang, CC: On the entire solutions of certain class of non-linear differential equations. J. Math. Anal. Appl. 33, 644-649 (1971)

7. Laine, I, Yang, CC: Clunie theorems for difference and q-difference polynomials. J. Lond. Math. Soc. 76(2), 556-566 (2007)

8. Laine, I, Yang, CC: Value distribution of difference polynomials. Proc. Jpn. Acad., Ser. A, Math. Sci. 83, 148-151 (2007)

9. Chen, ZX: Growth and zeros of meromorphic solution of some linear difference equations. J. Math. Anal. Appl. 373, 235-241 (2011)

10. Yang, CC, Laine, I: On analogies between nonlinear difference and differential equations. Proc. Jpn. Acad., Ser. A, Math. Sci. 86, 10-14 (2010)

11. Clunie, J: On integral and meromorphic functions. J. Lond. Math. Soc. 37, 17-27 (1962)

12. Yang, CC: A generalization of a theorem of P. Montel on entire functions. Proc. Am. Math. Soc. 26, 332-334 (1970)

13. Yang, CC, Li, P: On the transcendental solutions of a certain type of nonlinear differential equations. Arch. Math. 82, 442-448 (2004)

14. Chiang, YM, Feng, SJ: On the Nevanlinna characteristic of $f(z+\eta)$ and difference equations in the complex plane. Ramanujan J. 16, 105-129 (2008)

15. Halburd, RG, Korhonen, RJ: Difference analogue of the lemma on logarithmic derivative with applications to difference equations. J. Math. Anal. Appl. 314, 477-487 (2006)

16. Halburd, RG, Korhonen, RJ: Nevanlinna theory for the difference operator. Ann. Acad. Sci. Fenn.. Math. 31, 463-478 (2006)

17. Liu, K, Qi, XG: Meromorphic solutions of q-shift difference equations. Ann. Pol. Math. 101, 215-225 (2011)

18. Halburd, RG, Korhonen, RJ, Tohge, K: Holomorphic curves with shift-invariant hyperplane preimages. Trans. Am. Math. Soc. (to appear). arXiv:0903.3236

19. Bank, BS, Gundersen, GG, Laine, I: Meromorphic solutions of the Riccati differential equation. Ann. Acad. Sci. Fenn., Ser. A 1 Math. 6, 369-398 (1981)

10.1186/1029-242X-2014-63

Cite this article as: Liu et al.: Entire solutions of certain type of difference equations. Journal of Inequalities and Applications 2014, 2014:63

\section{Submit your manuscript to a SpringerOpen ${ }^{\circ}$ journal and benefit from:}

- Convenient online submission

- Rigorous peer review

Immediate publication on acceptance

Open access: articles freely available online

- High visibility within the field

- Retaining the copyright to your article 